\title{
Enhanced Susceptibility to 5-Fluorouracil in Human Colon Cancer Cells by Silencing of GRP78
}

\author{
SEUNGPIL YUN ${ }^{1}$, YONG-SEOK HAN ${ }^{2,3}$, JUN HEE LEE ${ }^{4}$, SANGMIN KIM ${ }^{1}$ and SANG HUN LEE ${ }^{2,3}$ \\ ${ }^{1}$ Neuroregeneration and Stem Cell Programs, Institute for Cell Engineering, \\ Department of Neurology, The Johns Hopkins University School of Medicine, Baltimore, MD, U.S.A.; \\ ${ }^{2}$ Medical Science Research Institute, Soonchunhyang University, Seoul Hospital, Seoul, Republic of Korea; \\ ${ }^{3}$ Department of Medical Bioscience, Soonchunhyang University, Asan, Republic of Korea; \\ ${ }^{4}$ Department of Pharmacology and Toxicology, \\ University of Alabama at Birmingham School of Medicine, Birmingham, AL, U.S.A.
}

\begin{abstract}
Background: Glucose-regulated protein (GRP78), also known as immunoglobulin heavy chain binding protein and as heat shock $70 \mathrm{kDa}$ protein 5, is present in the endoplasmic reticulum (ER) membrane. GRP78 is generally expressed at low concentrations, but is increased by physiological stress. GRP78 is thought to protect against tissue or organ damage under pathological conditions, such as neurotoxic stress, myocardial infarction, or arteriosclerosis. In addition, in tumors, GRP78 expression is much higher than in normal tissues. Furthermore, high levels of GRP78 expression have been shown to increase the risk of malignancy and metastasis in prostate and colon cancer. Because both anticancer drugs and down-regulation of GRP78 expression inhibit cancer progression and growth, we hypothesized that down-regulation of GRP78 expression might lead to enhanced susceptibility of cancer cells to cytotoxic action of 5fluorouracil (5-FU). Materials and Methods: GRP78 expression was suppressed in LoVo colon cancer cells by utilizing small-interfering RNA (si-GRP78), and the cells were subsequently used to study the antiproliferative and anticancer effects of 5-FU treatment. The signaling pathways responsible for the increase of LoVo cell susceptibility to 5-FU treatment after exposure to GRP78 siRNA were determined by western blot. Results: GRP78 silencing significantly inhibited cell viability and increased apoptosis of LoVo cells. Furthermore, combined treatment with 5-FU and GRP78 siRNA for $12 \mathrm{~h}$ reduced cell viability, and increased apoptosis and generation
\end{abstract}

Correspondence to: Sang Hun Lee, Ph.D., Soonchunhyang Medical Science Research Institute, Soonchunhyang University, Seoul Hospital, 59, Daesagwan-ro (657 Hannam-dong), Yongsan-gu, Seoul140-887, Republic of Korea. Tel: +82 027099029, Fax: +82 027925812,e-mail: ykckss1114@nate.com,jhlee0407@sch.ac.kr

Key Words: Colon cancer, 5-FU, GRP78, ER stress, apoptosis. reactive oxygen species more strongly than either of the two treatments applied separately. In order to examine the role of ER stress in increased susceptibility of LoVo cells to 5-FU after pretreatment with GRP78 siRNA, we analyzed expression levels of ER stress marker proteins, such as phosphorylated protein kinase-like endoplasmic reticulum kinase (PERK), phosphorylated eukaryotic initiation factor 2 alpha (eIF2 $\alpha$ ), activating transcription factor 4 (ATF4), phosphorylated inositol-requiring enzyme 1 alpha (IRE1 $\alpha)$, phosphorylated p38, and C/EBP homologous protein (CHOP). Treatment with 5-FU alone increased the expression of ER stress marker proteins, whereas combined exposure to both 5-FU and GRP78 siRNA led to an even stronger effect on these markers. Similar to the pattern of modulation of ER stress protein expression, the levels of apoptosis-related proteins were also more strongly affected by combined exposure to 5-FU and GRP78 siRNA than by single treatments. In particular, expression of Bcl-2-associated X protein (BAX), cleaved caspase-3, and cleaved poly (ADP-ribose) polymerase 1 (PARP1) were increased, whereas the expression of $B$-cell lymphoma 2 (BCL2) was reduced by these treatments. Conclusion: GRP78 silencing and incubation with 5-FU have synergistic effects on the inhibition of LoVo colon cancer cell growth via the induction of ER stress-dependent apoptosis.

The endoplasmic reticulum (ER) is a membranous organelle in eukaryotic cells involved in cellular homeostasis and apoptosis of cells in response to numerous physiological stresses (1). Consequently, dysregulation of ER functions has been correlated with various pathological states of cancer cells (1). Malignant tumors cause hypoxia, nutrient depletion, and generation of excess reactive oxygen species (ROS) of the surrounding microenvironment of the cell, perturbing the function of ER (2-4). Such cellular stress conditions activate the unfolded protein response (UPR) in $\mathrm{ER}$, which is known to enhance cell survival by preventing 
cells from entering apoptotic or senescence programs induced by cancer-related dysfunction (5). Likewise, there are various proteins in the ER membrane that act to prevent cell death caused by cancer. Specifically, previous studies suggested a potential role of glucose-regulated protein (GRP) 78 gene in UPR-related anti-apoptotic processes: i) GRP78 was found to be consistantly overexpressed in human colorectal cancer (CRC); ii) human CRC cells with overexpression of GRP78 exhibited increased survival, proliferation, and metastasis (6). In addition, it has been demonstrated that GRP78 promotes resistance to chemotherapeutic agents $(7,8)$. In light of these findings, further studies addressing the role of GRP78 in cancer progression are necessary. In particular, increasing attention has been paid to the precise relationship between GRP78 and $\mathrm{CRC}$, which is one of the most commonly diagnosed cancers in the world, with 1.3 million new cases diagnosed annually (9). Colon cancer and its prognosis is generally unfavorable because CRC is particularly malignant and resistant to many therapeutic drugs, mostly due to the loss of apoptosis signaling (9). However, despite its malignancy, CRC and its mechanisms have not been sufficiently elucidated. Previous studies have documented a potential role of the pro-survival property of GRP78 in inducing tumor growth and, possibly, in conferring drug resistance in colon cancer cells (10). In some tumors, overexpression of the GRP78 gene accompanied an increase in tumorigenicity and drug resistance (11-13). As a result, it has been hypothesized that overexpression of GRP78 might be involved in the pathogenesis of colorectal carcinomas (14). Association of GRP78 protein functions with colon cancer was specifically studied in cell lines (10). However, the role of GRP78 in the regulation of apoptosis has never been studied in LoVo colon cancer cells as far as we are aware. In the present study, we therefore sought to fill this gap in the literature and elucidated the relationship between down-regulation of GRP78 and cell death in human LoVo colon cancer cells.

Chemotherapy continues to play a vital role as an adjuvant therapy in CRC, and 5-fluorouracil (5-FU) remains a drug of choice for patients with CRC (15). Specifically, 5-FU kills cancer cells by increasing ROS generation and inducing ER stress, promoting, in turn, cancer cell apoptosis (16-19). However, there have been many cases in which drugresistant tumor cells emerged after 5-FU treatment (17-19). Because resistance to the induction of apoptosis is a prominent cause of chemotherapy failure, strengthening proapoptotic action by using combination therapy is currently the goal of many novel treatments. Recently, a potential correlation between 5-FU effects and GRP78 activity has been of interest in the literature. Because GRP78 is involved in the cell survival pathway, and GRP78 overexpression is implicated in drug resistance, a potentially useful treatment would enhance the 5-FU apoptotic pathway activity by concomitantly down-regulating GRP78. Such a combination of genetic regulation of a pro-survival protein with a drug treatment that induces cellular apoptosis may be a more effective mode of CRC therapy, as CRC tumors exhibit particularly high levels of drug resistance and poor prognosis. Thus, in response to increased attention on combination anticancer therapies, our study aimed to affect cancer cell viability at both the genetic and pharmacological levels. Furthermore, we hypothesized that we would see a synergistic impact on the induction of apoptosis in LoVo CRC cell line.

\section{Materials and Methods}

Cell culture. LoVo human colon cancer cells were obtained from the American Type Culture Collection (Manassas, VA, USA). The cells were maintained in Dulbecco's modified Eagle's medium (DMEM; $4.5 \mathrm{~g} / \mathrm{l}$ glucose) supplemented with $10 \%$ fetal calf serum, L-glutamine, and antibiotics (Biological Industries, Beit Haemek, Israel) at $37^{\circ} \mathrm{C}$ and in humidified atmosphere of $95 \% \mathrm{O}_{2}$ and $5 \%$ $\mathrm{CO}_{2}$ in an incubator.

Transfection with siRNA. LoVo cells were grown to $70 \%$ confluence in $60-\mathrm{mm}$ culture plates and washed twice with phosphate-buffered saline (PBS). The cells were transfected for $48 \mathrm{~h}$ with $100 \mathrm{nM}$ SMART pool siRNAs specific to GRP78 mRNA or non-targeting siRNA (scramble siRNA) using Lipofectamine 2000 reagent (Thermo Fisher Scientific, Waltham, MA, USA) in serum-free DMEM, according to the manufacturer's protocols.

Cell viability assay. LoVo cells were cultured in a 96-well culture plate with 5,000 cells/well. LoVo cells were then exposed to siGRP78 or scrambled si-RNA. 5-FU (Sigma-Aldrich, St. Louis, MO, USA) was dissolved in dimethyl sulfoxide, filter-sterilized using a $0.45-\mu \mathrm{m}$ pore filter (Sartorius Biotech $\mathrm{GmbH}$, Göttingen, Germany), and stored as $500 \mathrm{mg} / \mathrm{ml}$ stock at $4^{\circ} \mathrm{C}$ until use. The cells were treated with $25 \mu \mathrm{M} \mathrm{5-FU}$ at $37^{\circ} \mathrm{C}$ for $48 \mathrm{~h}$, and then cell survival assay was performed. The assay used was a modified 3-(4,5dimethylthiazol-2-yl)-2,5-diphenyltetrazolium bromide (MTT) assay based on the conversion of the tetrazolium salt to formazan by mitochondrial NAD $(\mathrm{P}) \mathrm{H}$-dependent oxidoreductases. Formazan levels were quantified by measuring the absorbance at $575 \mathrm{~nm}$ by using a microplate reader (BMG labtech, Allmendgrün, Ortenberg Germany).

Propidium iodide/annexin V flow cytometric analysis. In order to examine the level of apoptosis of LoVo cells transfected with scrambled si-RNA or si-GRP78 in the presence or absence of 5-FU, cells were stained with annexin V-fluorescein isothiocyanate (FITC) and propidium iodide (PI) (De Novo Software, Los Angeles, CA, USA) and evaluated using a Cyflow Cube 8 FACS (Sysmex Partec, Görlitz, Germany). Data were analyzed using standard FSC Express software (De Novo Software).

Dihydroethidium staining. Dihydroethidium (DHE; Sigma-Aldrich) was used to measure superoxide anion levels in LoVo cells transfected with scrambled si-RNA or si-GRP78 in the presence or absence of 5-FU. The cells were incubated with $10 \mu \mathrm{M}$ DHE for $30 \mathrm{~min}$ at $37^{\circ} \mathrm{C}$. After washing with PBS three times, samples were 
analyzed by flow cytometry (Cyflow Cube 8 FACS; Sysmex Partec). Data were analyzed using standard FSC Express software (De Novo Software).

Western blot analysis. Total cellular protein of LoVo cells was extracted using RIPA lysis buffer (Thermo Fisher Scientific). Cell lysate containing $20 \mu \mathrm{g}$ of protein was separated via $10 \%$ sodium dodecyl sulfate-polyacrylamide gel electrophoresis and transferred to nitrocellulose membranes for antibody probing. After washing with TBST (10 Mm Tris- $\mathrm{HCl}, 150 \mathrm{mM} \mathrm{NaCl}, 0.05 \%$ Tween-20, $\mathrm{pH}$ 7.6), membranes were blocked with 5\% skimmed milk for $2 \mathrm{~h}$ and then incubated with primary antibodies against GRP78, phosphorylated-protein kinase-like endoplasmic reticulum kinase (PERK), total PERK, phosphorylated-eukaryotic initiation factor 2 alpha (eIF2 $\alpha$ ), total eIF2 $\alpha$, activating transcription factor 4 (ATF4), phosphorylated-inositol-requiring enzyme 1 alpha (IRE1 $\alpha$ ), total IRE1 $\alpha$, phosphorylated-c-Jun N-terminal kinases (JNK), total JNK, phosphorylated-p38, total p38, C/EBP homologous protein (CHOP), B-cell lymphoma 2 (BCL2), BCL2-associated X protein (BAX), cleaved caspase-3, cleaved poly (ADP-ribose) polymerase 1 (PARP1), and $\beta$-actin (all from Santa Cruz Biotechnology, Dallas, TX, USA). After the incubation of the membranes with peroxidaseconjugated secondary antibodies (Santa Cruz Biotechnology), bands were detected using enhanced chemiluminescence reagents (Amersham Biosciences, Little Chalfont, UK) in a dark room.

Statistical analysis. Results are expressed as the mean \pm standard error of the mean (SEM). All experiments were analyzed by one-way analysis of variance. Comparisons of results in three or more groups were made using the Bonferroni-Dunn test. Differences were considered statistically significant when the $p$-value was less than 0.05 .

\section{Results}

GRP78 silencing enhanced 5-FU-induced cell death of LoVo colon cancer cells. Before evaluating potential synergistic effects of 5-FU and GPR78 down-regulation on cancer cell viability, the efficiency of GRP78 silencing was verified by immunoblot analyses, which demonstrated a significant reduction of GRP78 expression in cells transfected with siGRP78 (Figure 1A). To assess the effects of 5-FU and GRP78 silencing applied individually and in combination on LoVo cell viability and apoptosis, MTT and annexin V/PI apoptosis assays using flow cytometry were performed. As shown in Figure 1B, treatment with 5-FU reduced the viability of LoVo cells. Combined action of 5-FU and GPR78 siRNA reduced cell viability even further (Figure 1B). Indeed, treatment with 5-FU significantly increased the percentage of early and late apoptotic cells to $23.83 \%$ compared to that in control cells $(3.11 \%)$. In addition, in cells transfected with si-GRP78, early and late apoptotic cells comprised only $5.37 \%$. The percentage of apoptotic cells was much higher in cell cultures transfected with siGRP78 and treated with 5-FU (42.72\%) (Figure 1C and D). These results suggest that GPR78 down-regulation in combination with the treatment with $5-\mathrm{FU}$ has a strong anticancer effect in a colon cancer model.
GRP78 silencing enhanced 5-FU-induced accumulation of ROS. In order to determine the relationship between GRP78 expression and 5-FU-induced generation of ROS, we examined the latter using DHE, a small-molecule fluorescent ROS probe specific for $\mathrm{O}_{2}^{-}$(20), under silencing of GRP78 expression in colon cancer cells. The reaction between $\mathrm{O}_{2}{ }^{-}$ and DHE generates a highly specific red fluorescent product, 2-hydroxyethidium [2-OH-E(+)], shifting its excitation and emission peak wavelengths from 350 and $400 \mathrm{~nm}$ to 518 and $605 \mathrm{~nm}$, respectively $(21,22)$. LoVo cells were pretransfected with GRP78 siRNA and then exposed to 5-FU for $12 \mathrm{~h}$. DHE fluorescence intensity at 518 and $605 \mathrm{~nm}$ was then quantified using flow cytometry. Treatment with either 5-FU or si-GRP78 alone increased DHE-positive cell numbers (Figure 2A and B). In addition, combined treatment with 5-FU and si-GRP78 led to a stronger increase in the number of DHE-positive cells than in the case of both individual treatments (Figure 2A and $\mathrm{B}$ ). These results suggest that down-regulation of GRP78 expression has a synergistic effect on 5-FU-induced ROS accumulation.

GRP78 silencing enhanced 5-FU-induced ER stress. It is known that during ER stress, the expression of ER stressrelated proteins changes in ER membrane due to the activity of PERK, IRE1a, and ATF (23). Thus, we assumed that combined exposure to 5-FU treatment and GRP78 silencing would strongly increase expression of ER stress marker proteins in colon cancer cells. As shown in Figure 3A and B, our assumption was correct, and the exposure to the combination of 5-FU and si-GRP78 increased PERK phosphorylation, eIF2 $\alpha$ phosphorylation, and ATF4 expression stronger than either of the two treatments applied individually. Furthermore, as shown in Figure 4A and B, combined treatment with 5-FU and si-GRP78 also increased IRE1 $\alpha$ phosphorylation, p38 phosphorylation, and CHOP expression more potently than did 5-FU or si-GRP78 alone. These results suggest that GRP78 silencing further enhances the consequences of ER stress caused by 5 -FU.

GRP78 silencing enhanced 5-FU-induced apoptosis of human colon cancer cells. As demonstrated above, GRP78 silencing increased the number of apoptotic colon cancer cells after treatment with 5-FU. However, because cell viability and apoptosis assay data cannot provide clues to the mechanistic explanations for the sensitization to 5-FU action in the background of GRP78 silencing, we sought to examine changes in the expression of several apoptosisrelated proteins in colon cancer cells in greater detail. We reveal that incubation with $5-\mathrm{FU}$ led to a decrease in the expression of anti-apoptotic protein BCL2, whereas expression levels of the pro-apoptotic proteins BAX, cleaved caspase-3, and cleaved PARP1 were increased by that treatment (Figure $5 \mathrm{~A}$ and $\mathrm{B}$ ). In addition, we found that 
A
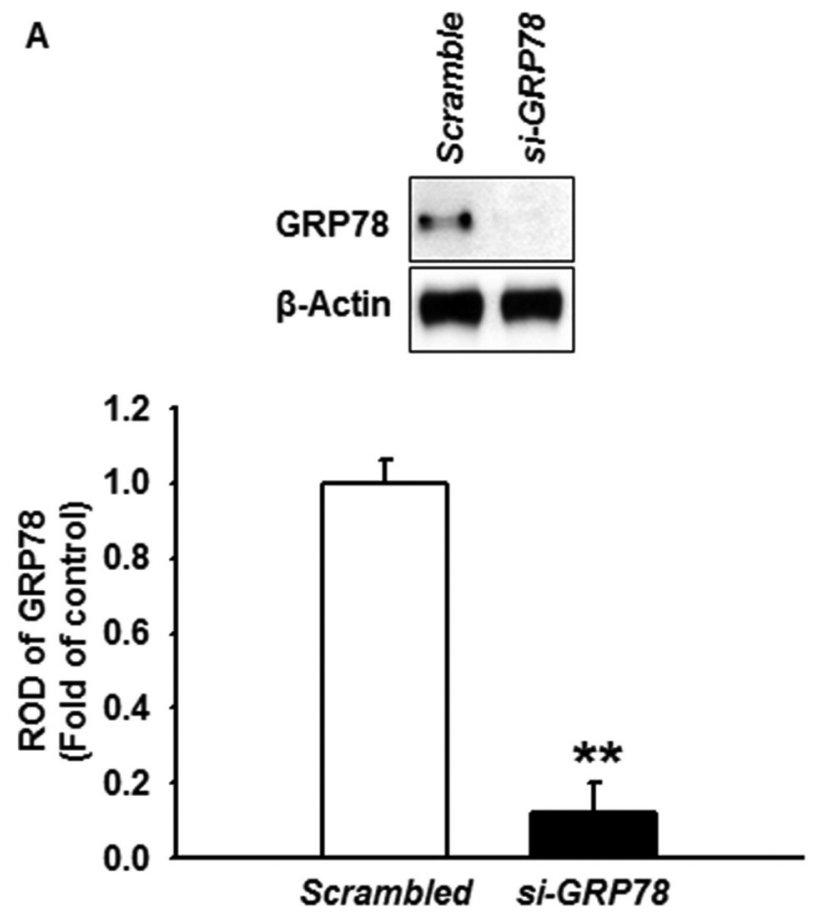

B

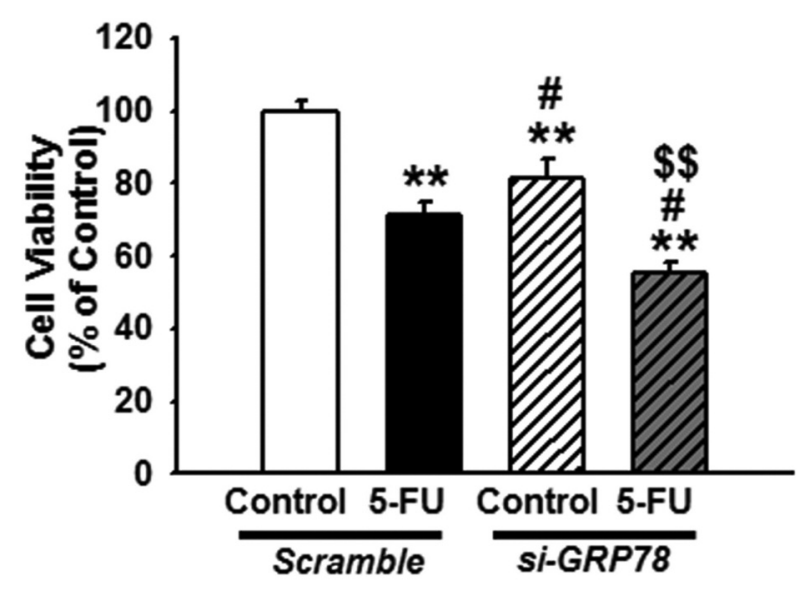

C

$\overline{\mathbf{L}}$
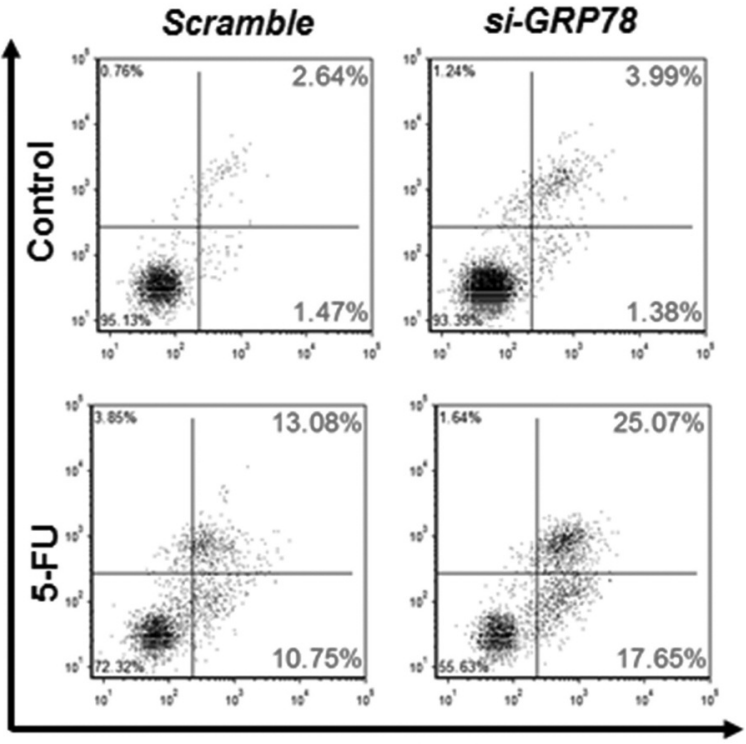

Annexin V
D

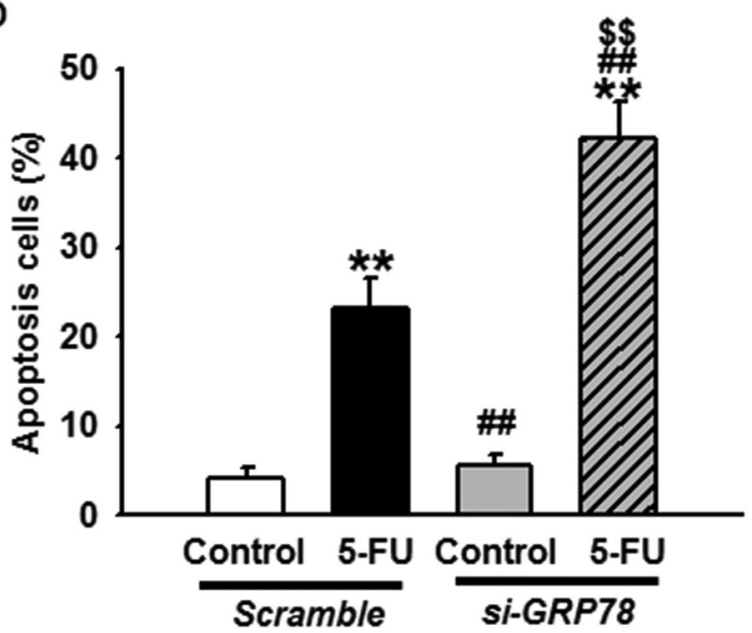

Figure 1. Effect of glucose-regulated protein (GRP78) silencing on 5-fluorouracil-induced LoVo colon cancer cell death. A: Efficacy of reduction of basal GRP78 level by GRP78 siRNA (si-GRP78) was determined by western blot. Cells were pretreated with si-GRP78 or non-targeting scrambled siRNA for $48 \mathrm{~h}$. Total protein was extracted and immunoblotted with an antibody to GRP78. The amount of $\beta$-actin was used as an internal loading control. Bar graph illustrates mean ( \pm SEM) normalized GRP78 levels in analyzed samples. B: LoVo cells were pre-transfected with si-GRP78 for $48 \mathrm{~h}$ and then treated with $25 \mu M$ 5-fluorouracil (5-FU) for 48 h. Cell viability was measured using a modified MTT assay. Data are expressed as the mean $\pm S E M$. C: Apoptosis of LoVo cells was measured using propidium iodide (PI)/annexin V staining and flow cytometry. PI/annexin V double-negative cells were considered live cells, PI-negative/annexin V-positive cells were considered early apoptotic cells, and PI/annexin V double-positive cells were considered late apoptotic cells. D: Standard quantification of PI/annexin V-positive apoptotic cells. Data are expressed as the mean \pm SEM. Statistical significance is indicated as follows: ** $p<0.01 \mathrm{vs.} \mathrm{control,}{ }^{\#} p<0.05$ and ${ }^{\# \#} p<0.01 \mathrm{vs}$. scrambled siRNA+5-FU, $\$ p<0.01$ vs. si-GRP78+5-FU.

combined treatment with si-GRP78 and 5-FU caused more pronounced changes in the above-mentioned parameters than those observed in single treatment groups (Figure 5A and $\mathrm{B})$.

\section{Discussion}

Anticancer drugs and GRP78 are known to modulate cancer progression and growth. In the present study, we showed that 
A

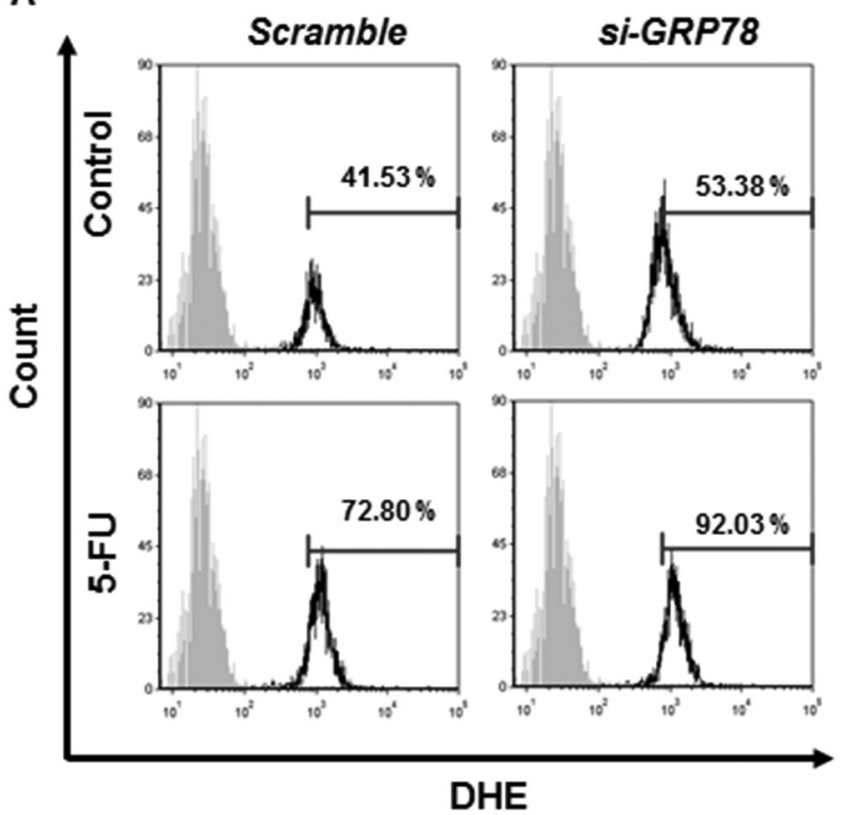

B

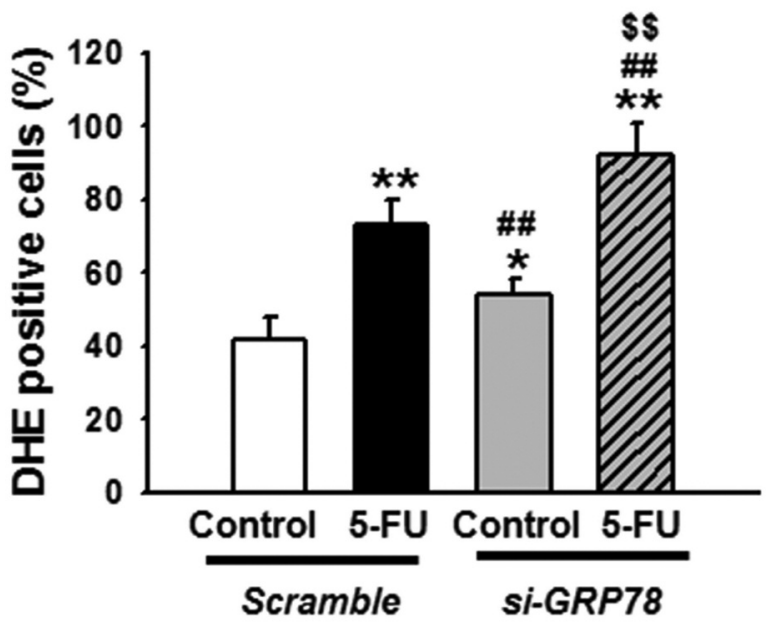

Figure 2. Glucose-regulated protein (GRP78) silencing enhanced 5-fluorouracil-induced reactive oxygen species accumulation in LoVo colon cancer cells. LoVo cells were pre-transfected with GRP78 siRNA (si-GRP78) or non-targeting scrambled siRNA 48 h before the treatment with $25 \mu M$ fluorouracil (5-FU) for 48 h. Reactive oxygen species accumulation was measured using dihydroethidium (DHE) staining. A: Representative flow cytometry image. B: Quantitative analysis of the percentage of DHE-positive cells by flow cytometry. Data are expressed as the mean \pm SEM. Statistical significance is indicated as follows: $* p<0.05$ and ${ }^{* *} p<0.01 \mathrm{vs}$. control, \#\# ${ }^{*}<0.01 \mathrm{vs}$. scrambled siRNA $+5-F U, \$ \$ p<0.01$ vs. si-GRp $78+5-F U$.

exposure to 5-FU and GRP78 silencing have synergistic effects on the inhibition of LoVo colon cancer cell growth mediated by the induction of ER stress-dependent apoptosis.

After lung and liver cancer, CRC is the third most prevalent form of malignant cancer and the fourth leading contributor to cancer-related deaths in the world (24). It has been estimated that approximately 1.2 million new cases and 600.000 deaths per year occur as a result of CRC (24). Currently, the only viable treatment available to treat patients with CRC is surgical resection followed by chemotherapy or radiotherapy (24). Specifically, the most widely utilized chemotherapeutic drug to treat CRC is 5-FU. It is a pyrimidine-based analog that is frequently used for treatment of various malignancies, including gastrointestinal, breast, head and neck, ovarian cancer, in addition to CRC (15). However, the extent of 5-FU-induced cancer cell apoptosis is limited due to the developing resistance to the drug and its use is restricted because of harmful side-effects (25). In particular, it has been suggested that GRP78 counteracts the cytotoxic action of chemical therapy with 5 -FU by protecting cells from 5-FU-induced apoptosis and promoting resistance to this drug $(10,26)$. As a result, there has been an increasing interest to synergistic combination therapies, whereby multiple targets would be simultaneously affected to enhance effects of anticancer drugs. In our study, we aimed to discover a potential synergistic therapeutic intervention that would comprise a cytotoxic treatment $(5-\mathrm{FU})$ together with the modulation at a genetic level (GRP78 silencing) to stop proliferation of human colon cancer cells. Our hypothesis was that because 5-FU is a potent anticancer drug, and $G R P 78$ overexpression renders cancer cells resistant to 5-FU, down-regulation of GRP78 would enhance pro-apoptotic, anticancer effects of 5-FU.

Given the relationship between GRP78 and 5-FU, we postulated that silencing GRP78 in cancer cells may potentiate the activity of drug-induced pro-apoptotic pathway, that would potentially lead to cancer cell death due to increased susceptibility to apoptosis. Thus, our study aimed to test whether silencing GRP78 expression was able to enhance 5-FU-induced inhibition of cancer cell proliferation in LoVo cells. The results obtained supported our initial hypothesis, as we demonstrate that a combination of 5-FU and GRP78 silencing led to a higher level of CRC cell death than that observed when either 5-FU or GRP78 silencing were applied separately (Figure 1). Statistical analysis suggested an additive effect of GRP78 gene silencing to the cytotoxic action of 5-FU chemical treatment (Figure1). Nevertheless, further mechanistic studies will be 

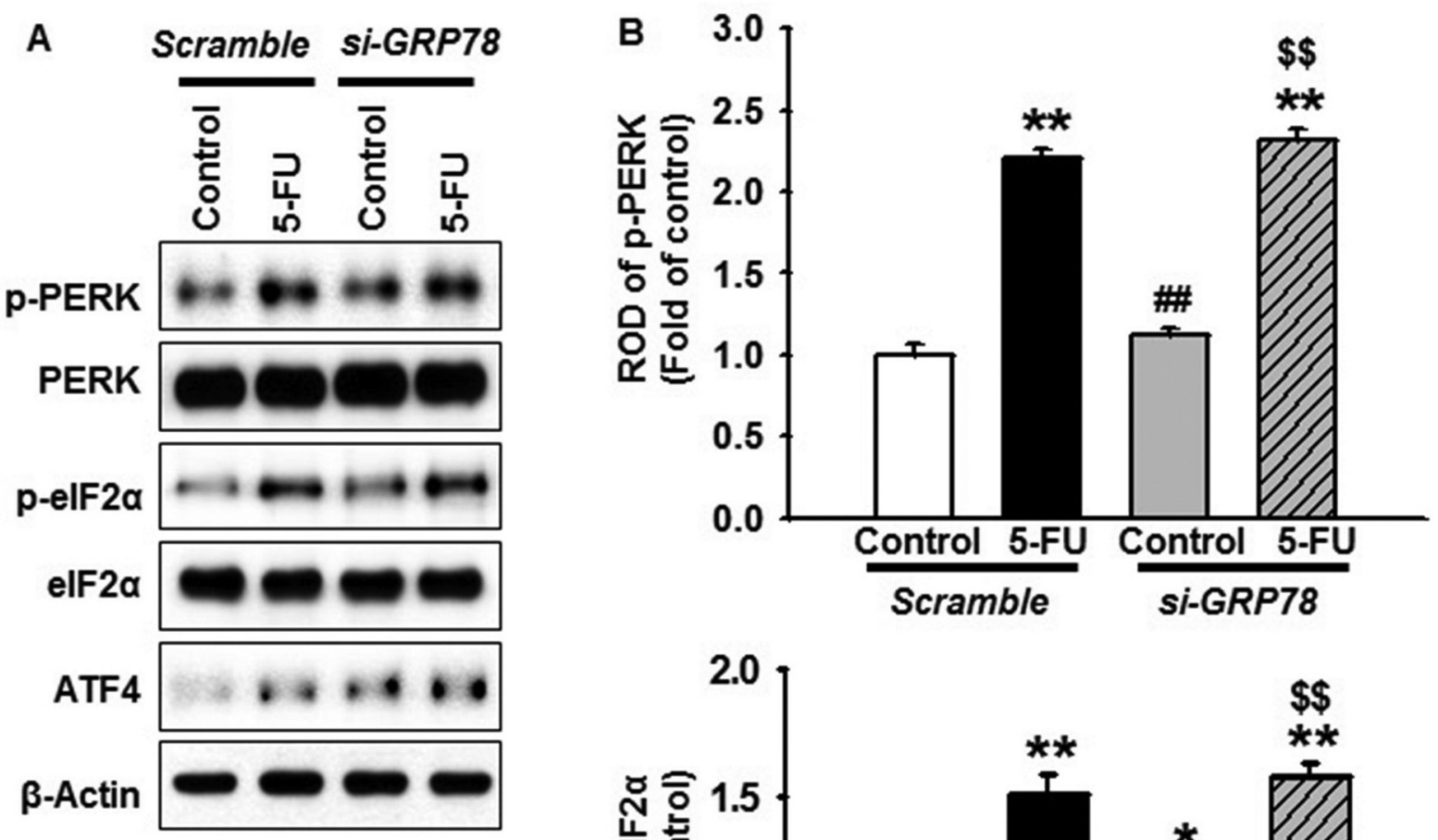

Figure 3. Glucose-regulated protein (GRP78) silencing enhanced 5-fluorouracil (5-FU)-induced endoplasmic reticulum stress via protein kinase-like endoplasmic reticulum kinase (PERK)-related signaling in LoVo colon cancer cells. LoVo cells were pre-transfected with GRP78 siRNA (si-GRP78) or non-targeting scrambled siRNA $48 \mathrm{~h}$ before the treatment with $25 \mu M$ 5-FU for $48 \mathrm{~h}$. A: Total protein was extracted and immunoblotted with antibodies against phosphorylated PERK, total PERK, phosphorylated eukaryotic initiation factor 2 alpha (eIF2 $\alpha$ ), total eIF $2 \alpha$, and activating transcription factor 4 (ATF4). Amounts of $\beta$-actin were used as internal loading controls. B: Bar graph illustrates mean $( \pm$ SEM) normalized levels of phosphorylated PERK, phosphorylated eIF2 $\alpha$, and ATF4 in analyzed samples. Statistical significance is indicated as follows: ${ }^{*} p<0.05$ and ${ }^{* *} p<0.01$ vs. control, ${ }^{\#} p<0.05$ and ${ }^{\# \#} p<0.01$ vs. scrambled siRNA +5-FU, ${ }^{\$} \$<<0.01$ vs. si-GRP78+5-FU.
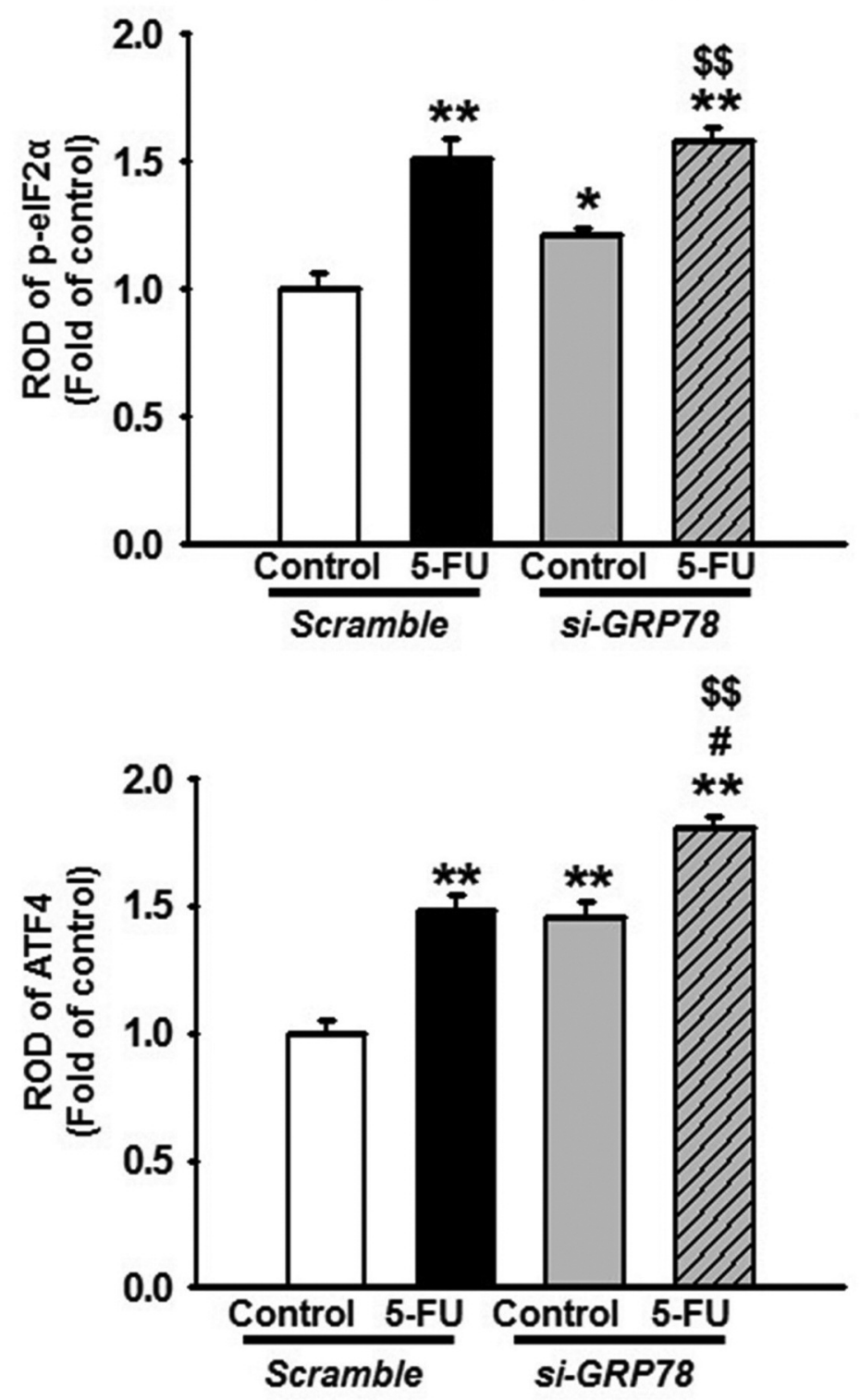
A

Scramble si-GRP78
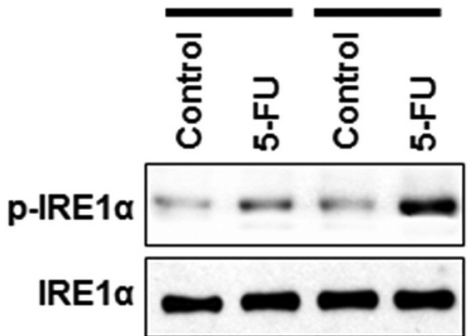

p-JNK

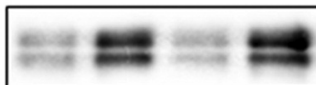

JNK

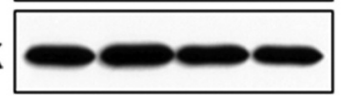

в
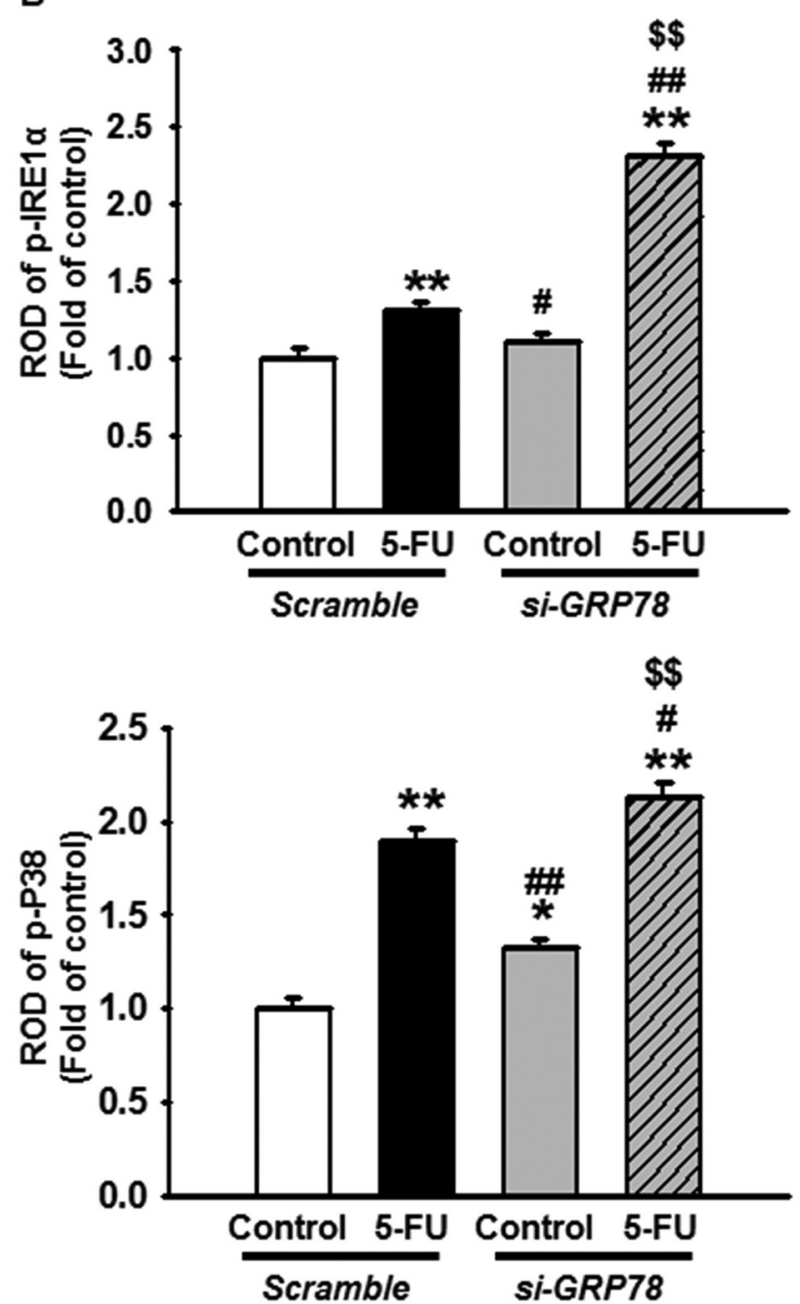

Scramble si-GRP78
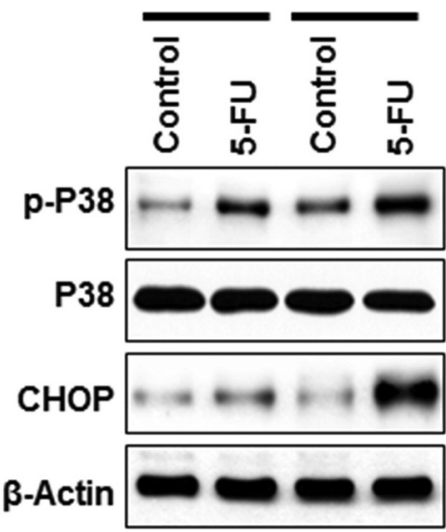

$\beta$-Actin
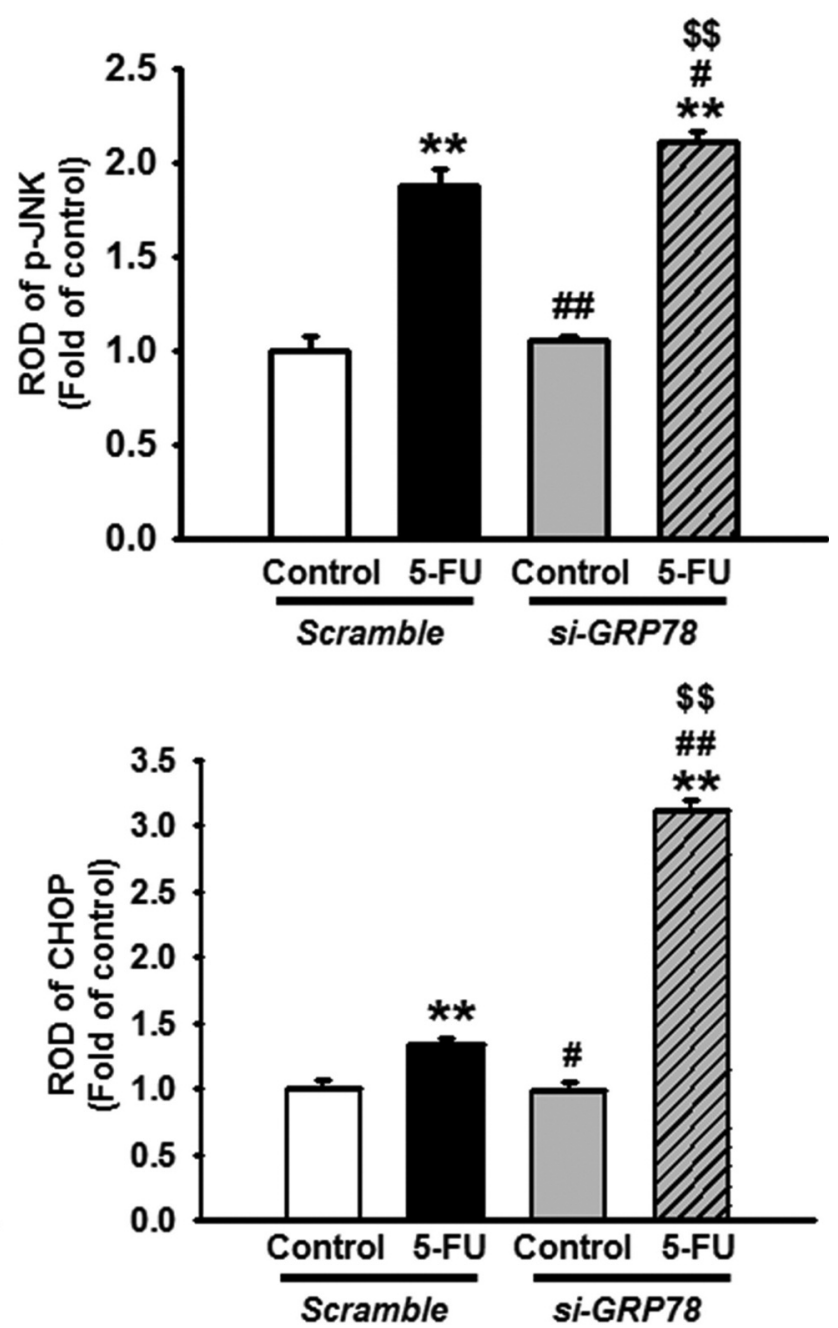

Figure 4. Glucose-regulated protein (GRP78) silencing enhanced 5-fluorouracil (5-FU)-induced endoplasmic reticulum stress via inositol-requiring enzyme 1 alpha (IRE1 $\alpha$ )-related signaling in LoVo colon cancer cells. LoVo cells were pre-transfected with GRP78 siRNA (si-GRP78) or nontargeting scrambled siRNA 48 h before the treatment with $25 \mu M$ 5-FU for 48 h. A: Total protein was extracted and immunoblotted with antibodies against phosphorylated IREI $\alpha$, total IRE1 $\alpha$, phosphorylated $p 38$, total $p 38$, and C/EBP homologous protein (CHOP). Amounts of $\beta$-actin were used as internal loading controls. B: Bar graph illustrates mean $( \pm$ SEM) normalized levels of phosphorylated IRE1 $\alpha$, phosphorylated p38, and CHOP in analyzed samples. Statistical significance is indicated as follows: ${ }^{*} p<0.05$ and ${ }^{* *} p<0.01 \mathrm{vs}$. control, ${ }^{\#} p<0.05$ and ${ }^{\# \#} p<0.01 \mathrm{vs}$. scrambled siRNA $+5-F U, \$ \$ p<0.01$ vs. si-GRP78+5-FU. 
A

Scramble si-GRP78

Scramble si-GRP78
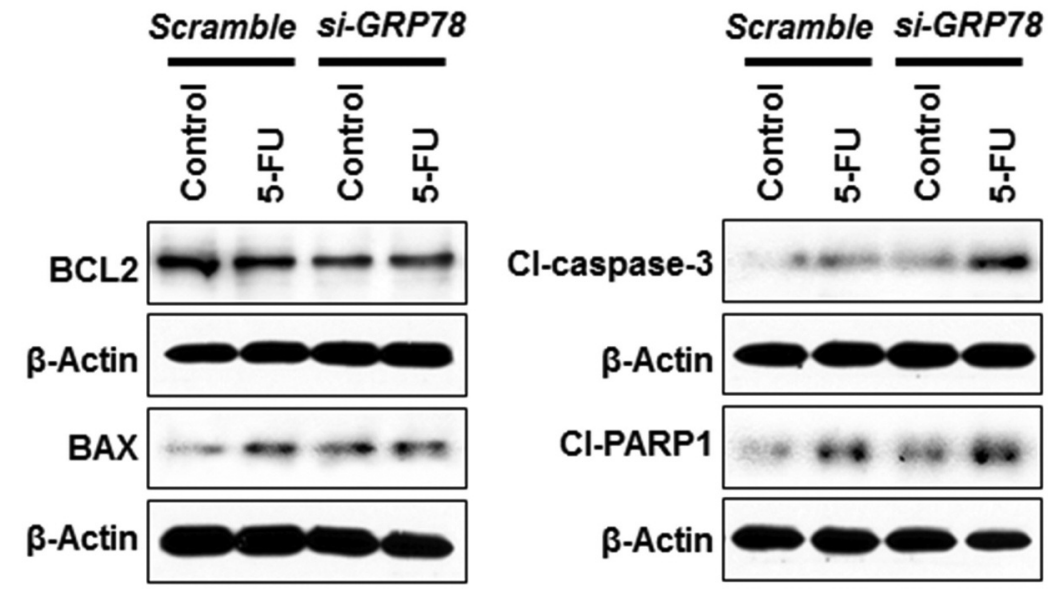

B
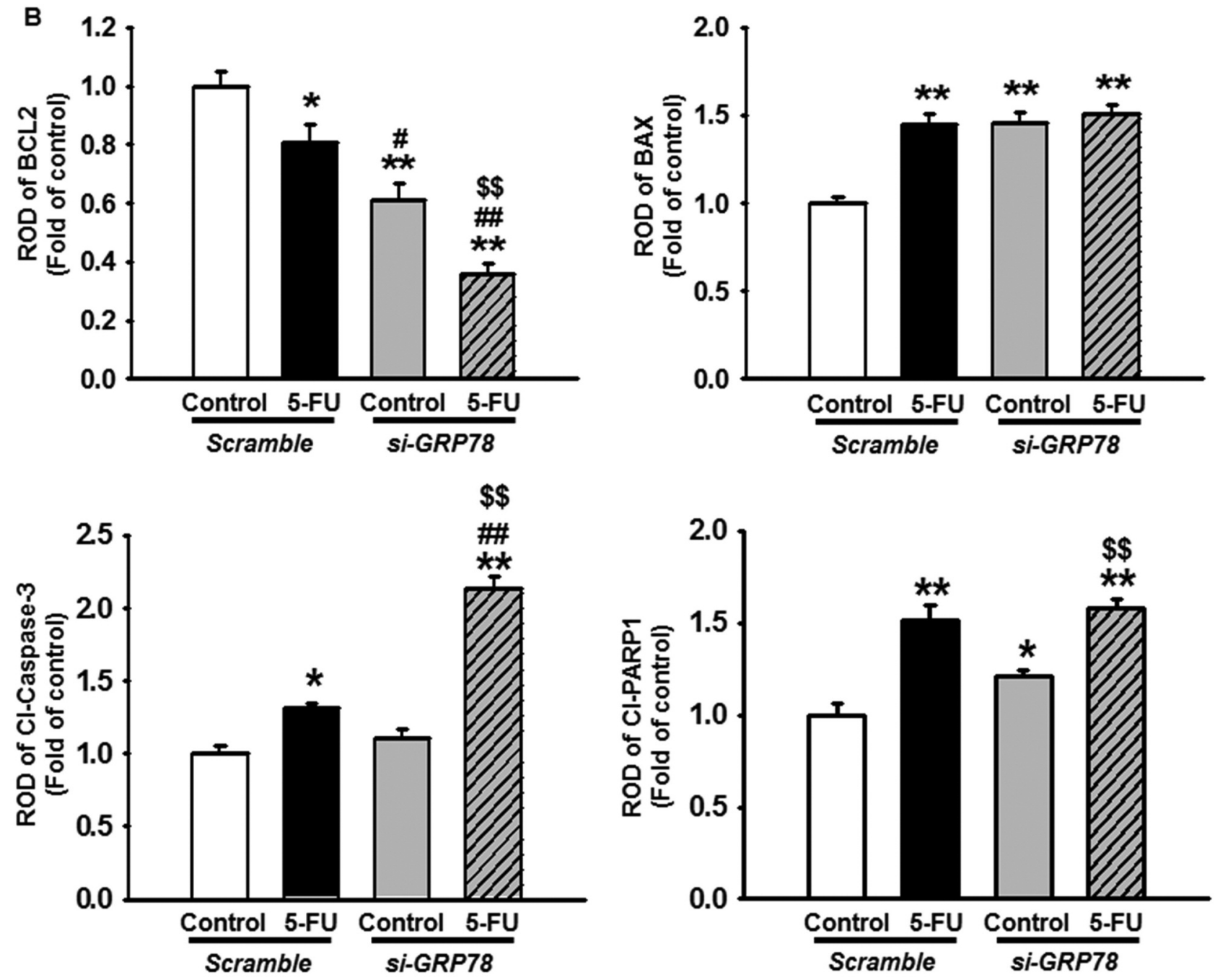

Figure 5. Effect of glucose-regulated protein (GRP78) silencing on 5-fluorouracil (5-FU)-induced LoVo colon cancer cell apoptosis. LoVo cells were pre-transfected with GRP78 siRNA (si-GRP78) 48 h before the treatment with $25 \mu M$ 5-FU. A: Total protein was extracted and immunoblotted with antibodies against B-cell lymphoma 2 (BCL2), BCL2-associated X protein (BAX), cleaved (Cl) caspase-3, and cleaved poly (ADP-ribose) polymerase 1 (Cl-PARP1). The amount of $\beta$-actin was used as internal loading control. B: Bar graph illustrates the mean ( \pm SEM) normalized levels of BCL2, BAX, cleaved caspase-3, and PARP1 in analyzed samples. Statistical significance is indicated as follows: $* p<0.05$ and $* * p<0.01$ vs. control, ${ }^{\#} p<0.05$ and ${ }^{\# \#} p<0.01$ vs. scrambled siRNA +5-FU, $\$ \$ p<0.01$ vs. si-GRP78+5-FU. 
critical to establish the precise pathway(s) that link the effects of treatment with 5-FU and GRP78 silencing. Several studies have demonstrated that 5-FU induces cancer cell death by increasing ROS generation, thereby producing ER stress and promoting apoptosis in cancer cells $(4,16,27)$. ROS generation could be an important tool of cancer therapy due to its potential ability to inflict oxidative damage to vital biological molecules, including DNA, lipids, and proteins, altering cellular integrity and initiating apoptosis (28-31). In LoVo colon cancer cells, 5-FU negatively affected CRC growth by increasing ROS generation, causing ER stress, which, in turn, induced cell apoptosis. In addition, higher levels of GRP78 production in cancer cells is known to produce ER-stress tolerance because GRP78 is a calciumbinding molecular chaperone located in the ER that works to maintain ER homeostasis and suppresses stress-induced apoptosis $(32,33)$. Thus, our results demonstrated that in combination with 5-FU, down-regulation of GRP78 in LoVo cancer cells led to higher generation of ROS and ER stress level (Figures 2-4). Specifically, the expression of ER stressrelated proteins, such as PERK, IRE1a, and ATF became higher. Pro-apoptotic proteins BAX, cleaved caspase-3, and cleaved-PARP1 were increased in cells treated at both genetic and protein levels, suggesting that silencing of GRP78 enhances 5-FU-induced cell apoptosis. Therefore, our results strongly suggest that a combination of 5-FU treatment and down-regulation of GRP78 successfully enhances ROS generation, ER stress, and eventual apoptosis of LoVo cancer cell. Further studies are needed to ascertain whether alterations in apoptosis caused by 5-FU are mediated by known cell senescence mechanistic pathways specifically in LoVo cells.

Taken together, the results of our study revealed a potentially better approach for chemotherapy by establishing knockdown of GRP78 enhances cancer cell apoptosis, ROS generation, and activation of the ER stress signaling pathway by 5 -FU. Given that cells may become resistant to increased ROS levels, the induction of ER stress in human colon cancer cells by a novel combination therapy that diminishes GRP78 expression and up-regulates ROS generation by 5FU may not only have implications for understanding individual anticancer effects of these two treatments, but also pave the way to more effective cancer cure and better patient outcomes.

\section{Conflicts of Interest}

The Authors have no conflicts of interest to declare with regards to this study.

\section{Acknowledgements}

This study was supported by the Soonchunhyang University Research Fund, a National Research Foundation grant funded by the
Korean government (NRF-2016R1D1A3B01007727), and a grant from the Korean Health Technology R\&D Project, Ministry of Health and Welfare, Republic of Korea (HI14C2253). The funders had no role in study design, data collection or analysis, decision to publish, or preparation of the article.

\section{References}

1 Moenner M, Pluquet O, Bouchecareilh $\mathrm{M}$ and Chevet E: Integrated endoplasmic reticulum stress responses in cancer. Cancer Res 67: 10631-10634, 2007.

2 Schroder M and Kaufman RJ: The mammalian unfolded protein response. Annu Rev Biochem 74: 739-789, 2005.

3 Lee AS: GRP78 induction in cancer: therapeutic and prognostic implications. Cancer Res 67: 3496-3499, 2007.

4 Appierto V, Tiberio P, Villani MG, Cavadini E and Formelli F: $P L A B$ induction in fenretinide-induced apoptosis of ovarian cancer cells occurs via a ROS-dependent mechanism involving ER stress and JNK activation. Carcinogenesis 30: 824-831, 2009.

5 Xu C, Bailly-Maitre B and Reed JC: Endoplasmic reticulum stress: cell life and death decisions. J Clin Invest 115: 26562664, 2005.

6 Shuda M, Kondoh N, Imazeki N, Tanaka K, Okada T, Mori K, Hada A, Arai M, Wakatsuki T, Matsubara O, Yamamoto N and Yamamoto M: Activation of the ATF6, XBP1 and GRP78 genes in human hepatocellular carcinoma: a possible involvement of the ER stress pathway in hepatocarcinogenesis. J Hepatol 38 : 605-614, 2003.

7 Chatterjee S, Cheng MF, Berger RB, Berger SJ and Berger NA: Effect of inhibitors of poly(ADP-ribose) polymerase on the induction of GRP78 and subsequent development of resistance to etoposide. Cancer Res 55: 868-873, 1995.

8 Belfi CA, Chatterjee S, Gosky DM, Berger SJ and Berger NA: Increased sensitivity of human colon cancer cells to DNA crosslinking agents after GRP78 up-regulation. Biochem Biophys Res Commun 257: 361-368, 1999.

9 Torre LA, Bray F, Siegel RL, Ferlay J, Lortet-Tieulent J and Jemal A: Global cancer statistics, 2012. CA Cancer J Clin 65: 87-108, 2015.

10 Hardy B, Raiter A, Yakimov M, Vilkin A and Niv Y: Colon cancer cells expressing cell surface GRP78 as a marker for reduced tumorigenicity. Cell Oncol 35: 345-354, 2012.

11 Al-Rawashdeh FY, Scriven P, Cameron IC, Vergani PV and Wyld L: Unfolded protein response activation contributes to chemoresistance in hepatocellular carcinoma. Eur J Gastroenterol Hepatol 22: 1099-1105, 2010.

12 de Ridder G, Ray R, Misra UK and Pizzo SV: Modulation of the unfolded protein response by GRP78 in prostate cancer. Methods Enzymol 489: 245-257, 2011.

13 Daneshmand S, Quek ML, Lin E, Lee C, Cote RJ, Hawes D, Cai J, Groshen S, Lieskovsky G, Skinner DG, Lee AS and Pinski J: Glucose-regulated protein $G R P 78$ is up-regulated in prostate cancer and correlates with recurrence and survival. Hum Pathol 38: 1547-1552, 2007.

14 Takahashi H, Wang JP, Zheng HC, Masuda S and Takano Y: Overexpression of GRP78 and GRP94 is involved in colorectal carcinogenesis. Histol Histopathol 26: 663-671, 2011.

15 White RE: Irradiation stents: a clever and maybe costly, fusion. Lancet Oncol 15: 546-547, 2014. 
16 Chinta SJ, Rane A, Poksay KS, Bredesen DE andersen JK and Rao RV: Coupling endoplasmic reticulum stress to the cell death program in dopaminergic cells: effect of paraquat. Neuromolecular Med 10: 333-342, 2008.

17 Berberat PO, Dambrauskas Z, Gulbinas A, Giese T, Giese N, Kunzli B, Autschbach F, Meuer S, Buchler MW and Friess H: Inhibition of heme oxygenase-1 increases responsiveness of pancreatic cancer cells to anticancer treatment. Clin Cancer Res 11: 3790-3798, 2005.

18 Kim SK, Yang JW, Kim MR, Roh SH, Kim HG, Lee KY, Jeong HG and Kang KW: Increased expression of NRF2/AREdependent anti-oxidant proteins in tamoxifen-resistant breast cancer cells. Free Radic Biol Med 45: 537-546, 2008.

19 Was H, Dulak J and Jozkowicz A: Heme oxygenase-1 in tumor biology and therapy. Curr Drug Targets 11: 1551-1570, 2010.

20 Zhao H, Kalivendi S, Zhang H, Joseph J, Nithipatikom K, Vasquez-Vivar J and Kalyanaraman B: Superoxide reacts with hydroethidine but forms a fluorescent product that is distinctly different from ethidium: potential implications in intracellular fluorescence detection of superoxide. Free Radic Biol Med 34: 1359-1368, 2003.

21 Zhao H, Joseph J, Fales HM, Sokoloski EA, Levine RL, Vasquez-Vivar $\mathrm{J}$ and Kalyanaraman B: Detection and characterization of the product of hydroethidine and intracellular superoxide by HPLC and limitations of fluorescence. Proc Natl Acad Sci USA 102: 5727-5732, 2005.

22 Zielonka J, Srinivasan S, Hardy M, Ouari O, Lopez M, VasquezVivar J, Avadhani NG and Kalyanaraman B: Cytochrome cmediated oxidation of hydroethidine and mito-hydroethidine in mitochondria: identification of homo- and heterodimers. Free Radic Biol Med 44: 835-846, 2008.

23 Jakobsen CH, Storvold GL, Bremseth H, Follestad T, Sand K, Mack M, Olsen KS, Lundemo AG, Iversen JG, Krokan HE and Schonberg SA: DHA induces ER stress and growth arrest in human colon cancer cells: associations with cholesterol and calcium homeostasis. J Lipid Res 49: 2089-2100, 2008.

24 Brenner H, Kloor M and Pox CP: Colorectal cancer. Lancet 383: 1490-1502, 2014.

25 Longley DB and Johnston PG: Molecular mechanisms of drug resistance. J Pathol 205: 275-292, 2005.
26 Benatti P, Basile V, Dolfini D, Belluti S, Tomei M and Imbriano C: NF-Y loss triggers p53 stabilization and apoptosis in HPV18positive cells by affecting E6 transcription. Oncotarget 7: 4590145915, 2016.

27 Verfaillie T, Rubio N, Garg AD, Bultynck G, Rizzuto R, Decuypere JP, Piette J, Linehan C, Gupta S, Samali A and Agostinis P: PERK is required at the ER-mitochondrial contact sites to convey apoptosis after ROS-based ER stress. Cell Death Differ 19: 1880-1891, 2012.

28 Dewaele M, Maes H and Agostinis P: ROS-mediated mechanisms of autophagy stimulation and their relevance in cancer therapy. Autophagy 6: 838-854, 2010.

29 Nicolau-Galmes F, Asumendi A, Alonso-Tejerina E, Perez-Yarza G, Jangi SM, Gardeazabal J, Arroyo-Berdugo Y, Careaga JM, Diaz-Ramon JL, Apraiz A and Boyano MD: Terfenadine induces apoptosis and autophagy in melanoma cells through ROSdependent and -independent mechanisms. Apoptosis 16: 12531267, 2011.

30 Bellot GL, Liu D and Pervaiz S: ROS, autophagy, mitochondria and cancer: RAS, The hidden master? Mitochondrion 13: 155$162,2013$.

31 Yoon S, Woo SU, Kang JH, Kim K, Kwon MH, Park S, Shin HJ, Gwak HS and Chwae YJ: STAT3 transcriptional factor activated by reactive oxygen species induces IL6 in starvationinduced autophagy of cancer cells. Autophagy 6: 1125-1138, 2010.

32 Kim JK, Kang KA, Piao MJ, Ryu YS, Han X, Fernando PM, Oh MC, Park JE, Shilnikova K, Boo SJ, Na SY, Jeong YJ, Jeong SU and Hyun JW: Endoplasmic reticulum stress induces 5fluorouracil resistance in human colon cancer cells. Environ Toxicol Pharmacol 44: 128-133, 2016.

33 Pyrko P, Schonthal AH, Hofman FM, Chen TC and Lee AS: The unfolded protein response regulator GRP78/BiP as a novel target for increasing chemosensitivity in malignant gliomas. Cancer Res 67: 9809-9816, 2007.

Received April 9, 2017

Revised April 21, 2017

Accepted April 25, 2017 